\title{
Kneeling assessment after Anterior Cruciate ligament reconstruction using Bone-Patellar Tendon-Bone autograft versus hamstring autograft
}

Fahad Al Hulaibi ( $\nabla$ dr.fahad.hu@gmail.com)

Dammam Medical Complex https://orcid.org/0000-0002-3369-8148

Zainab Al Eid

King Fahad Hospital

Saad Altaher

Dammam Medical Complex

Abdulaziz Albarrak

Dammam Medical Complex

\section{Zaid Alzaid}

King Faisal University

Research article

Keywords: Anterior cruciate ligament, Bone-Patellar Tendon-Bone graft, Hamstring autograft, kneeling

Posted Date: May 4th, 2020

DOl: https://doi.org/10.21203/rs.3.rs-24903/v1

License: (a) This work is licensed under a Creative Commons Attribution 4.0 International License. Read Full License 


\section{Abstract \\ Background}

ACL reconstruction surgeries is a common surgery that aim to provide stability and return functionality post ACL injury. Commonly, bone patellar tendon-bone (BPTB) and hamstring tendon autografts are used for the reconstruction. The choice of optimal autograft remains debatable to achieve satisfactory outcomes for various activities after reconstruction, including kneeling as widely needed, especially in prayers among Muslims. Kneeling with considerable ranges of motion and the minimum level of pain is still under rigorous investigations to choose proper autograft.

\section{Purpose}

Hereby, we compare kneeling outcomes post-operatively using bone-patellar tendon-bone autografts with hamstring autografts for ACL reconstruction.

\section{Methods}

A retrospective cohort study; Level of evidence (III) has enrolled. Adult patients underwent primary ACL reconstruction using either BPTB or HS autografts after they consented at a single institution with a minimum of 1-year as follow-up post-operatively were reviewed for assessment of the surgery's outcomes in regards of kneeling pain and subjective assessment measures using International Knee Documentation Committee (IKDC) evaluation system.

\section{Results}

A total of Thirty-two males were met our inclusion criteria. Analysis of data provided that 12 patients were in the BTB group and 20 patients in the HS group. In all patients of our study, ACL rupture was sustained during participation in athletic activity regardless the type of sport with $60.8 \%$ patients were affected of left knee. After follow-up of 1-year, clinical evaluation of anterior drawer test postoperatively resulted with Two patients in HS group were positive. There was no statistically significant difference between scores of two groups when comparing patient-reported outcome measures, with mean IKDC scores of 68.5 and 62.55 in the BTB and HS groups, respectively. Interestingly, insignificant statistical difference observed in both groups in regard of kneeling pain in the BTB group when compared with the HS group.

\section{Conclusion}

The findings of our study have shown that Arthroscopic ACL reconstruction using either hamstring autograft or bone patellar tendon autograft ends with equally reasonable satisfactory outcomes, with an 
insignificant difference in kneeling pain between both groups.

\section{Background}

An anterior cruciate ligament ( $A C L)$ is a common knee ligamentous injury that causing instability with an incidence of 0.38 per 1000 each year in the united states [1]. The ACL reconstruction surgeries aim to provide stability and return functionality and expected to prevent injuries of the meniscus or leading to degenerative changes such as osteoarthritis [2].

It is a common practice that bone patellar tendon-bone (BPTB) and hamstring tendon autografts are used for the reconstruction. Bone patellar tendon-bone (BPTB) autograft was first described by Jones in 1960, which became popular by Erickson until the late 1970s [3]. However, this methodology of reconstruction was associated with various post-operative issues such as harvesting site morbidity and stiffness. A systematic review conducted from the USA by Monaghan et al. has shown an increase in long-term anterior knee pain, kneeling pain, and higher rates of osteoarthritis were noted with BPTB graft use [4]. This motivated surgeons to look for alternatives with reasonable outcomes for their patients as at the beginning of the 1990s [3], a new methodology was introduced to reconstruct the anterior cruciate ligament through two hamstring tendons (HT): gracilis and semitendinosus.

The choice of optimal autograft either BPTB or HS autograft remains controversial to achieve satisfactory outcomes for various activities with a considerable range of motion and minimal anterior knee pain after the reconstruction [5]. It is attributed to the advantages and disadvantages each autograft has and the absence of clear evidence-based guidelines that recommend one autograft in favor of the other [6]. Ideally, the graft used for ACL reconstruction should reproduce the anatomic and biomechanical properties of the native ligament, and using each autograft is debatable as each one has its characteristics. Nevertheless, a long-term prospective study that compared patellar tendon versus semitendinosus tendon autografts for anterior cruciate ligament reconstruction shows no significant differences between the groups in donor-site morbidity [7]. However, most of the surgeons in our area avoid BPTB graft of ACL reconstruction as they claimed that it could cause anterior knee pain and difficulty in kneeling, especially in Muslims who need hyperflexion during praying multiple times a day [8].

Therefore, our study aimed to measure the functional outcomes and kneeling ability of patients whose underwent ACL reconstruction by BPTB autograft among Saudi Arabian patients and compare the results with hamstring autograft. The primary endpoint of this project was to aid the surgeon in making a correct decision with their patients with minimal post-operative anterior knee pain experienced by patients. A secondary objective was to describe the severity of the pain during a range of daily activities, but particularly during kneeling, which is essential knee activity among Muslim people to perform prayers.

\section{Materials And Methods}


Our Retrospective cohort study enrolled adult patients whose underwent $A C L$ reconstruction with autologous bone-patellar tendon graft (BPTB) and hamstring autograft that met inclusion criteria. Pediatric age group younger than 18 years old or patients with any associated ligamentous injuries that require surgery, previous meniscectomy, or meniscal injury requiring more than one-third meniscectomy at the time of reconstruction were excluded.

Graft choice between BPTB and HS autograft was decided after a thorough discussion of the risks and benefits of each one between the patient and the surgeon. Surgeries conducted at a single tertiary hospital in Saudi Arabia by three fellowship-trained sports surgeons. A standard rehabilitation protocol was prescribed to all patients by the physiotherapy department after the surgery, regardless of the ACL graft that they received.

At mean follow-up duration with a minimum 1-year post-reconstruction, patients who were willing to participate interviewed and inquired with using the International Knee Documentation Committee evaluation (IKDC 2000) score to measure the functional outcomes of the operated knee. The demographic data collected, including patients' age, sex, BMI affected knee, type of graft used, and duration since the injury until surgery has been performed. Thus, Kneeling pain was assessed by asking the patient to kneel on a carpeted surface for approximately 1 minute and to note the presence of pain. Intensity of pain was graded from 0 to 10 , with 0 being no pain and 10 being extremely severe. Pain scales were categorized as following: mild pain (0-3 points); moderate pain (4-7 points); and severe pain (8-10 points) [9]. To achieve our study's purpose, moderate and severe are the categories that indicate the existence of anterior knee pain. Furthermore, anterior drawer tests were performed before and after the surgery to evaluate the knee stability.

The data was analyzed using SPSS 23 version (Statistical Package for the Social Sciences; Chicago, IL). Descriptive statistics were conducted for the patient's demographic and categorical data. Comparisons of frequency counts for categorical data were made with the chi-square test. Likewise, for data of continuous variables, the paired samples t-test was used. A $p$ value of $<0.05$ considered a significant Statistically.

Informed consent was taken from all participants after a thorough explanation of the study's rationale. Institutional review board (IRB) approval obtained before the commencement of our study.

\section{Results}

\section{Patient Characteristics}

A total of thirty-two males were included in our study, with 12 patients were in the BPTB group, and 20 patients in the HS group. There were no statistical differences between the groups regarding age as the majority presented in the late $20 \mathrm{~s}$ and body mass index of being morbidly obese in both groups (Table 1). 
However, there was a significant difference in duration of injury until the surgery has been performed between the two groups i.e., in HS group patients extend to be for almost two years in comparison to BPTB of less than one year, respectively. In all patients of our study, the ACL rupture sustained during participation of the athletic activity regardless of sport type. $60.8 \%$ of the patients were affected of the left knee.

\section{Clinical Evaluation}

Patients who were available after a follow-up of 1 year without sustained complications of instability or re-rupture have undergone a clinical assessment with anterior drawer test and compared with the findings that exist before the surgery. Two patients in the HS group were positive after 16 months postoperatively. Lachman's test findings were not documented for all patients (Table 2).

\section{Patient-Reported Outcomes measure}

We found no significant difference between groups when comparing patient-reported outcome measures, with mean IKDC scores of 68.5 and 62.55 in the BPTB and HS groups, respectively (Table 3 ). Interestingly, the insignificant statistical difference observed in both groups in regard to kneeling pain in the BPTB group when compared with the HS group. According to IKDC item 9c, ten patients in the BPTB group versus 18 in the HS group experienced mild pain during kneeling $(p=0.862)$.

\section{Discussion}

Choosing the optimal graft for ACL reconstruction is remaining a controversial topic, attributed to the various factors that confound to obtain the proper decision such as patient's age, sports activities, surgeon preference, and nature of the occupation. The most common used autografts that surgeons preferred to use for their patients are hamstring tendon (HS) or bone-patellar tendon-bone (BPTB) autografts reconstruct the ACL [10] The current evidence with the available literature is still debated regarding the optimal option without the agreement of the superiority of a particular graft. Some literature recommended the use of BPTB autograft for the advantages of healing and increase stability (assessed through KT-1000 arthrometer testing), negative pivot shift, and decreased risk for revision [11, 12 [. Nevertheless, there are recent literature published that recommend HS autograft due to the advantages of increased extension strength, decrease the incidence of anterior knee pain, and minimal donor site morbidity] 13,14[. Another published studies support the use of hamstring autograft for those patients that cannot tolerate anterior knee pain as they need to kneel in various daily living activities and elucidate the possibility of having a higher risk to develop long term anterior knee pain with BPTB autograft compared with HS $[4,8,15,16]$. Some studies suggested the injury or neuroma of the infrapatellar branch of the saphenous nerve is suggested to be the cause of this knee pain [32]. A 2-incision approach was used for patellar tendon harvest, which has been shown previously to decrease the kneeling pain [32]. Moreover, patients who are treated with BPTB had increased pain in the acute postoperative period when compared with those treated with HS [30]. 
A meta-analysis of 22 studies with a total of 1930 patients undergoing ACL reconstruction conducted by Xie et al., reported that patients treated with BPTB autograft had more significant long-term kneeling pain and anterior knee pain when compared with those treated with HS autografts [17]. Furthermore, Li et al. evaluated outcomes of ACL reconstruction among nine randomized controlled trials for 738 patients and found that BPTB autografts produced significant anterior knee and kneeling pain. ]18[

Therefore, we aimed in our study to investigate the long-term outcomes of BPTB autograft versus HS autograft for which producing a better impact on patient's activities. Interestingly, our study conducted among sample of muslim patients whom need kneeling as a major activity to perform prayers which should assist the surgeon to select the proper autograft of superior outcomes for the injuried patients. which might contribute to assist the surgeon in selecting the autograft with superior outcomes in the region. Hereby, the purpose of our study was obtained by the international knee documentation committee (IKDC) subjective knee evaluation form, which is one of the reliable instruments used commonly to determine results following various knee procedures, including ACL reconstructions. Thus, it is valid to measure knee symptoms as well. [19]

This measurement tool was used as a primary method for the collected sample to measure subjective satisfaction and kneeling pain postoperatively. We found interestingly in our result that insignificant differences between groups when comparing patient-reported outcome measures, with mean IKDC scores of 68.5 and 62.55 in the BPTB and HS groups, respectively. According to IKDC item 9c, ten patients in the BPTB group versus 18 in the HS group experienced mild pain during kneeling, which is reasonable. Overall postoperative results are satisfactory within each group in terms of IKDC subjective scores, activities of daily living when compared to pre-operative scores. Similar findings reported by Eriksson et al. which were consistent with majority of patients in the group $80 \%$ scored normal and minimal pain while $20 \%$ of patients scored abnormal IKDC grades [20]. Outcomes of our study demonstrates that both types of reconstruction are effective methods of restoring knee stability and producing satisfactory outcomes in means of post-operative anterior knee pain and kneel pain. There was insignificant statistical difference in the IKDC score between two groups after 1-year of follow-up [21].

To minimize the bias of our results, we reviewed magnetic resonance imaging (MRI) findings of contributed patients for this study who scored severe kneeling pain to investigate whether any confounding risk factors participated. Two patients had BPTB autograft with a result of 7, 10 scores of kneeling pain, respectively. It consisted of complete ACL tear associated with features of posterior lateral corner syndrome and complicated Baker's cyst preoperatively in their MRI report. Additionally, one patient autografted with hamstring scored 10 (the maximum intensity) had high-grade anterior cruciate ligament tear associated with Posterior horn of medial meniscus oblique tear. No distinguished findings existed among patients who scored mild-moderate pain. Therefore, there is a correlation between the severity and the MRI abnormality findings.

A recent short-term study by Laxdal et al. showed that insignificant clinical differences could be found between two groups as well [22]. Similar findings published a long time in 1994 by Corry et al. found that 
the two grafts did not differ in terms of clinical stability, range of motion, and general symptoms, which consistent with our study findings [23]. Similarly, Biau et al. conducted a meta-analysis of various studies concluded that insignificant differences between two grafts but recommended HS for patients who need jumping [24]. However, this might be attributed to the newer surgical techniques that performed, which leads to an insignificant difference in terms of complications. This perspective of the new surgical techniques should be taken into consideration and investigate extensively as BPTB is still a considerable option that would be advised for patients of certain ethnic origins and religions such as Muslims who need to kneel for prayer [25].

In addition, the potential muscle weakness following hamstring autograft for ACL reconstruction is concerned and investigated in many studies. Current literature results that surgeons need to balance adequate graft size and strength with the potential for donor-site morbidity when performing an ACL reconstruction with hamstring autografts. However, this aspect remains not conclusive yet as a hamstring function analysis by certain types of sports would be required to elucidate this issue, and it does not a concern aspect currently in our study [26[. Chee et al. conducted a meta-analysis recently that comparing the outcomes of BPTB grafts versus 4-strand hamstring autografts. They reported a significant negative effect in the aspects of anterior knee pain, kneeling pain, and extension deficit that did not support the use of BPTB grafts with favored the hamstring autograft rather than the BPTB graft for anterior knee pain and kneeling pain. Thus, it concluded that the 4-strand hamstring ACL reconstruction has comparable clinical results with the BPTB graft but with fewer postoperative complications [27[. However, other studies have found that an ACL reconstruction with a BPTB graft might be superior in aspects of stability and preferred to be used for the young athletic patient ]17, 28[. Therefore, both autografts are providing satisfactory outcomes in long-term means.

It is evident that ACL reconstruction surgeries performed by trained sport orthopedic surgeons have better outcomes than surgeries performed by general orthopods. In our study, there were three fellowship-trained sports orthopedic surgeons operated on all patients, which considered as a strength, but various surgical techniques performed, which might contribute to results bias. Although we enrolled a consecutive series of patients in our study to reduce the margin of selection bias, these patients were not randomized, which have been subject to selection bias. Thus, all patients involved were males and young with ACL rupture was sustained during participation in the athletic activity regardless the type of sport. At a 10-year followup, there were no gender differences regarding outcomes of failures, radiographic osteoarthritis, or overall IKDC scores [31]. Additionally, our study has been performed on a retrospective review basis, and as such, it carries inherent shortcomings. Finally, the sample size of patients is considered minimal compare to the original patients that have been operated due to loss of follow-up and strict inclusion \& exclusion criteria we had, which potentially introduced bias to our results and reduce power for statistical significance and increase margin errors of data analysis.

\section{Conclusion}


Findings of our study have shown that Arthroscopic ACL reconstruction using either hamstring autograft or bone patellar tendon autograft ended with equally reasonable satisfactory outcomes regarding return to functionality and activities of daily living with an insignificant difference in kneeling pain between both groups. However, further long-term prospective randomized clinical trial studies are warranted.

\section{Abbreviations}

ACL Anterior cruciate ligament

BPTB Bone-Patellar Tendon-Bone autograft

HS Hamstring autograft

IKDC International Knee Documentation Committee

\section{Declarations}

- Ethics approval and consent to participate:

All procedures performed in the study were under the ethical standards of the institution at which the studies were conducted, and the ethical approval obtained. Informed consent was obtained from all individual participants included in the study.

\section{- Availability of data and materials:}

The datasets used and/or analysed during the current study are available from the corresponding author on reasonable request.

- Competing interests:

There is no conflict of interest

\section{- Funding:}

The authors declare that they have no funding for this research

\section{- Authors' contributions:}

We thank Khalid alhumam, Mohammad Alarbash, Haya AlMousa, and Hissah AlTurki for helping us in interviewing patients and collecting data. Also, I would like to thank all patients who cooperate with us in this research.

\section{- Acknowledgements:}


We thank Khalid alhumam, Mohammad Alarbash, Haya AlMousa, and Hissah AlTurki for helping us in interviewing patients and collecting data.

\section{- Author information:}

- Fahad H. al Hulaibi; Senior registrar of orthopedic surgery, Dammam complex medical complex, he corresponding author. Dammam, Saudi Arabia. Focus on Sport medicine researches. +966501876716, fahad.hu@gmail.com

- Zainab Al Eid, Orthopedic resident at King Fahd Hospital Hufof. AlAhsa, Saudi Arabia, +966567025838, aleidzainab@gmail.com

- Saad Al Taher, Consultant Orthopedic, sport medicine, reconstruction and foot and ankle specialist. Focus on Sport medicine researches. Dammam, Saudi Arabia, +966506931535, SALTAHER@moh.gov.sa

- Abdulaziz Albarrak, Senior registrar, of orthopedic surgery Dammam complex medical complex. Focus on Arthroplasty researches. Dammam, Saudi Arabia, +966548515707, abdulazizalbarrak1212@gmail.com

- Zaid Alzaid, Medical intern at King Faisal university. Dammam, Saudi Arabia. Focus on Sport medicine researches.+966551415581, Zaidalzaid.za@gmail.com

\section{References}

1. Miyasaka KC, Daniel DM, Shore ML, Hirsham P. The incidence of knee ligament injuries in the general population. Am J Knee Surg (1991); 4:3-8.

2. Fithian DC, Paxton LW, Goltz DH. Fate of the anterior cruciate ligament-injured knee. Orthop Clin North Am (2002); 33:621-36.

3. Chambat, C. Guier, B. Sonnery-Cottet, J.-M. Fayard, M. Thaunat. The evolution of ACL reconstruction over the last fifty years. Int Orthop, 37 (2013), pp. 181-186.

4. Poehling- KL, Salem H, Ross KE, Secrist E, Ciccotti MC, Tjoumakaris F, et al. Long-Term Outcomes in Anterior Cruciate Ligament Reconstruction: A Systematic Review of Patellar Tendon Versus Hamstring Autografts. Orthopaedic Journal of Sports Medicine. (2017);5(6):2325967117709735.

5. Pinczewski LA, Lyman J, Salmon LJ, Russell VJ, Roe J, Linklater J. A 10-year comparison of anterior cruciate ligament reconstructions with hamstring tendon and patellar tendon autograft: A controlled, prospective trial. Am J Sports Med (2007); 35:564-574.

6. P. Spindler, J.E. Kuhn, K.B. Freedman, C.E. Matthews, R.S. Dittus, F.E. Harrell. Anterior cruciate ligament reconstruction autograft choice: bone-tendon-bone versus hamstring: does it really matter? A systematic review. Am J Sports Med, 32 (2004), pp. 1986-1995 
7. Lidén M, Ejerhed L, Sernert N, Laxdal G, Kartus J. Patellar tendon or semitendinosus tendon autografts for anterior cruciate ligament reconstruction: a prospective, randomized study with a 7Year follow-up. The American Journal of Sports Medicine. (2007);35(5):740-8.

8. Thompson S, Salmon L, Waller A, Linklater J, Roe J, Pinczewski L. Twenty-year outcomes of a longitudinal prospective evaluation of isolated endoscopic anterior cruciate ligament reconstruction with patellar tendon autografts. The American Journal of Sports Medicine. (2015); 43(9):2164-74.

9. Boonstra AM, Schiphorst Preuper HR, Balk GA, Stewart RE. Cut-off points for mild, moderate, and severe pain on the visual analogue scale for pain in patients with chronic musculoskeletal pain. Pain. 2014;155(12):2545e2550.

10. Vaishya R, Agarwal AK, Ingole S, Vijay V. Current trends in anterior cruciate ligament reconstruction: a review. Cureus. (2015);7:e378.

11. Katabi M, Djian P, Christel P. Anterior cruciate ligament reconstruction: patellar tendon autograft versus four-strand hamstring tendon autografts. A comparative study at one-year follow-up. Rev Chir Orthop Reparatrice Appar Mot. (2002);88:139-148.

12. Persson A, Fjeldsgaard K, Gjertsen JE, et al. Increased risk of revision with hamstring tendon grafts compared with patellar tendon grafts after anterior cruciate ligament reconstruction: a study of 12,643 patients from the Norwegian Cruciate Ligament Registry, 2004- 2012. Am J Sports Med. (2014);42:285-291.

13. Magnussen RA, Carey JL, Spindler KP. Does autograft choice determine intermediate-term outcome of ACL reconstruction? Knee Surg Sports Traumatol Arthrosc. (2011);19:462-472.

14. Maletis GB, Cameron SL, Tengan JJ, Burchette RJ. A prospective randomized study of anterior cruciate ligament reconstruction: a comparison of patellar tendon and quadruple-strand semitendinosus/gracilis tendons fixed with bioabsorbable interference screws. Am J Sports Med. (2007);35:384-394.

15. Romanini E, D'Angelo F, De Masi S, et al. Graft selection in arthroscopic anterior cruciate ligament reconstruction. J Orthop Traumatol. (2010);11:211-219.

16. West RV, Harner CD. Graft selection in anterior cruciate ligament reconstruction. J Am Acad Orthop Surg. (2005);13:197-207.

17. Xie X, Liu X, Chen Z, Yu Y, Peng S, Li Q. A meta-analysis of bonepatellar tendon-bone autograft versus four-strand hamstring tendon autograft for anterior cruciate ligament reconstruction. Knee. (2015); 22:100-110.

18. Li S, Chen Y, Lin Z, Cui W, Zhao J, Su W. A systematic review of randomized controlled clinical trials comparing hamstring autografts versus bone-patellar tendon-bone autografts for the reconstruction of the anterior cruciate ligament. Arch Orthop Trauma Surg. (2012);132: 1287-1297.

19. Greco, N. J., Anderson, A. F., Mann, B. J., Cole, B. J., Farr, J., Nissen, C. W., \& Irrgang, J. J. Responsiveness of the International Knee Documentation Committee subjective knee form in comparison to the Western Ontario and McMaster Universities Osteoarthritis Index, modified 
Cincinnati Knee Rating System, and Short Form 36 in patients with focal articular cartilage defects. The American journal of sports medicine (2010); 38(5), 891-902.

20. ErikssonK, Anderberg P, Hamberg P, Löfgren AC, Bredenberg M, Westman I, et al. A comparison of quadruple semitendinosus and patellar tendon grafts in reconstruction of the anterior cruciate ligament. J Bone Joint Surg Br (2001);83:348-54.

21. Beynnon BD, Johnson RJ, Fleming BC, et al. Anterior cruciate ligament replacement. Comparison of bone-patellar tendon-bone grafts with two-strand hamstring grafts; a prospective randomized study. J Bone Joint Surg Am (2002); 84: 1503-13.

22. Laxdal G, Sernert N, Ejerhed L, Karlsson J, Kartus JT. A prospective comparison of bone-patellar tendon-bone and hamstring tendon grafts for anterior cruciate ligament reconstruction in male patients. Knee Surg Sports Traumatol Arthrosc (2007);15:115-25.

23. Good L, Odensten M, Gillquist J. Sagittal knee stability after anterior cruciate ligament reconstruction with a patellar tendon strip. A two-year follow-up study. Am J Sports Med (1994);22:518-23.

24. Biau DJ, Tournoux C, Katsahian S, Schranz PJ, Nizard RS. Bone-patellar tendon-bone autografts versus hamstring autografts for reconstruction of anterior cruciate ligament: Meta-analysis. BMJ (2006);332:995-1001.

25. Biau DJ, Katsahian S, Kartus J, Harilainen A, Feller JA, Sajovic M, et al. Patellar tendon versus hamstring tendon autografts for reconstructing the anterior cruciate ligament: A meta-analysis based on individual patient data. Am J Sports Med (2009);37:2470-8.

26. Norimasa Nakamura, Shuji Horibe, Satoru Sasaki, Takuya Kitaguchi, Mituo Tagami, Tomoki Mitsuoka, Yukiyoshi Toritsuka, Masayuki Hamada, and Konsei Shino. Evaluation of Active Knee Flexion and Hamstring Strength After Anterior Cruciate Ligament Reconstruction Using Hamstring Tendons . The Journal of Arthroscopic and Related Surgery (2002); 18(6): 598-602.

27. Chee MY, Chen Y, Pearce $C J$, et al. Outcome of patellar tendon versus 4-strand hamstring tendon autografts for anterior cruciate ligament reconstruction: a systematic review and meta-analysis of prospective randomized trials. Arthroscopy. (2017);33(2):450e463.

28. Erickson BJ, Harris JD, Fillingham YA, et al. Anterior cruciate ligament reconstruction practice patterns by NFL and NCAA football team physicians. Arthroscopy. (2014);30(6):731e738.

29. Hui, Catherine, et al. "Fifteen-Year Outcome of Endoscopic Anterior Cruciate Ligament Reconstruction With Patellar Tendon Autograft for 'Isolated' Anterior Cruciate Ligament Tear." The American Journal of Sports Medicine, vol. 39, no. 1, Jan. 2011, pp. 89-98, doi:10.1177/0363546510379975.

30. Okoroha KR, Keller RA, Jung EK, Khalil L, Marshall N, Kolowich PA, Moutzouros V. Pain Assessment After Anterior Cruciate Ligament Reconstruction: Bone-Patellar Tendon-Bone Versus Hamstring Tendon Autograft. Orthop J Sports Med. 2016 Dec 20;4(12):2325967116674924. doi: 10.1177/2325967116674924. PMID: 28210646; PMCID: PMC5298558.

31. Pinczewski, Leo A., et al. "A 10-Year Comparison of Anterior Cruciate Ligament Reconstructions with Hamstring Tendon and Patellar Tendon Autograft: A Controlled, Prospective Trial." The American Journal of Sports Medicine, vol. 35, no. 4, Apr. 2007, pp. 564-574, doi:10.1177/0363546506296042. 
32. Kartus J, Ejerhed L, Sernert N, Brandsson S, Karlsson J. Comparison of traditional and subcutaneous patellar tendon harvest: a prospec- tive study of donor site-related problems after anterior cruciate liga- ment reconstruction using different graft harvesting techniques. Am J Sports Med. 2000;28(3):328-335.

\section{Tables}

Patient Characteristics (Table1)

\begin{tabular}{|c|c|c|c|}
\hline & $\begin{array}{c}\text { BPTB mean } \\
\pm(\text { SD) }\end{array}$ & HS mean $\pm($ SD) & $p$ value \\
\hline Male (n) & 12 & 20 & Constant. \\
\hline Age (years) & $28.3(6.38)$ & $29(5.75)$ & 0.484 \\
\hline BMI & $24.37(1.33)$ & $26.934(3.055)$ & 0.384 \\
\hline $\begin{array}{c}\text { Injury duration } \\
\text { (months) }\end{array}$ & $10.42(9.85)$ & $24.05(4.45)$ & 0.043 \\
\hline Affected Knee (R/L) & $4 / 8$ & $9 / 11$ & 0.393 \\
\hline
\end{tabular}

BPTB, bone-patellar tendon-bone; HS, hamstring autograft; BMI, Body mass index. $p$-Value $<0.5$ indicated statistical significance.

\section{Objective outcomes by clinical evaluation (Table2)}

\begin{tabular}{|c|c|c|}
\hline Graft & $\begin{array}{c}\text { Anterior drawer } \\
\text { positive test (Pre- op) }\end{array}$ & $\begin{array}{c}\text { Anterior drawer test } \\
\text { (Post- op) }\end{array}$ \\
\cline { 2 - 3 } & Result (N) & Result (N) \\
\hline BPTB & 11 & 0 \\
\hline HS & 20 & 2 \\
\hline
\end{tabular}

BPTB, bone-patellar tendon-bone; HS, hamstring autograft.

Subjective patient-outcomes reports (Table3)

\begin{tabular}{|l|l|l|l|}
\hline & BPTB (n=12) $^{\mathbf{1}}$ & HS (n= 20) $^{\mathbf{1}}$ & $p$ value $^{\mathbf{2}}$ \\
\hline IKDC score $^{3}$ & $68.50 / 87(78.75 \%)$ & $62.55 / 87(71.86 \%)$ & 0.719 \\
\hline Kneeling pain $^{4}$ & & & \\
& & & 0.862 \\
Severe & 1 & 1 & \\
Moderate & 1 & 1 & \\
$\quad$ Mild & 10 & 18 & \\
\hline
\end{tabular}

1. Values are presented as mean $\pm \mathrm{SD}$ or $\mathrm{n}(\%)$.

2. $p<.05$. BPTB, bone-patellar tendon-bone; HS, hamstring autograft.

3. IKDC, International Knee Documentation Committee.

4. Responses from item 9c of the IKDC subjective evaluation form. 